\title{
Dirac Oscillator in a Galilean Covariant Non-commutative Space
}

\author{
G.R. de Melo • M. de Montigny • P.J. Pompeia • \\ E.S. Santos
}

Received: 29 June 2012 / Accepted: 12 September 2012 / Published online: 28 September 2012

(C) Springer Science+Business Media New York 2012

\begin{abstract}
We study the Galilean Dirac oscillator in a non-commutative situation, with space-space and momentum-momentum non-commutativity. The wave equation is obtained via a 'Galilean covariant' approach, which consists in projecting the covariant equations from a $(4,1)$-dimensional manifold with light-cone coordinates, to a $(3,1)$-dimensional Galilean space-time. We obtain the exact wave functions and their energy levels for the plane and discuss the effects of non-commutativity.
\end{abstract}

Keywords Non-commutative geometry $\cdot$ Galilean covariance $\cdot$ Dirac equation

G.R. de Melo

Núcleo Interdisciplinar em Ciência, Engenharia e Tecnologia Centro de Ciências Exatas e Tecnológicas, Universidade Federal do Recôncavo da Bahia, Campus Universitário de Cruz das Almas 44380-000,

Cruz das Almas, Bahia, Brazil

e-mail: gmelo@ufrb.edu.br

M. de Montigny $(\bowtie)$

Theoretical Physics Institute, University of Alberta, Edmonton, Alberta, Canada T6G 2E1

e-mail: mdemonti@ualberta.ca

M. de Montigny

Faculté Saint-Jean, University of Alberta, Edmonton, Alberta, Canada T6C 4G9

P.J. Pompeia

Instituto de Fomento e Coordenação Industrial, Departamento de Ciência e Tecnologia Aeroespacial, Praça Marechal Eduardo Gomes, 50, Vila das Acácias, CEP 12.228-901, São José dos Campos, SP, Brazil

e-mail: pedropjp@ifi.cta.br

E.S. Santos

Instituto de Física, Universidade Federal da Bahia, 40210-340 Salvador, Bahia, Brazil

e-mail: esdras.santos@ufba.br 


\section{Introduction}

The purpose of this paper is to apply 'Galilean covariance' (a formulation of non-relativistic theory exploiting covariant equations in higher dimension) to study the non-relativistic Dirac oscillator in a non-commutative space. This paper is the direct continuation of our previous study of the Galilean covariant Duffin-Kemmer-Petiau (DKP) spin-zero oscillator in a noncommutative space [1]. The DKP equation is a first-order wave equation which describes spin-zero and spin-one particles (see Refs. [2-6]). An advantage of the DKP formalism is that it facilitates the systematic introduction of non-minimal couplings in quantum theories [7, 8]. Hereafter we consider an analogous non-minimal coupling on a non-commutative space for non-relativistic spin-half particles by using a Galilean version of the Dirac equation [9-11]. We describe non-commutative spaces along the lines of Snyder's early paper on discrete space-time [12]. Our main motivation stems from the connection between noncommutative coordinates and discrete space-time, with a view to applying Galilean invariant lattice models to condensed matter physics.

Even though Galilean kinematics is only an approximation of the relativistic kinematics, the structure of Galilean kinematics is more intricate than relativistic kinematics. For instance, the Galilean algebra admits a non-trivial central extension and projective representations, whereas the Poincaré algebra does not [13-16]. As mentioned in Ref. [17], we can construct representations of the Galilei algebra with three possible methods: (1) directly from the Galilei algebra, (2) from contractions of the Poincaré algebra with the same space-time dimension, or (3) from the Poincaré algebra in a space-time with one additional dimension. In this paper, we follow the third approach, which we refer to as 'Galilean covariance' because the equations are Lorentz covariant in the extended manifold. These equations become Galilean invariant after projection to the lower dimension. Our motivation is that this covariant approach provides one more unifying feature of field theory models. Indeed, particle physics (with Poincaré kinematics) and condensed matter physics (with Galilean kinematics) share many tools of quantum field theory (e.g. gauge invariance, spontaneous symmetry breaking, Goldstone bosons), but the Galilean kinematics does not admit a metric structure [18]. However, since the Galilean Lie algebra is a subalgebra of the Poincaré Lie algebra if one more space-like dimension is added, we can achieve 'Galilean covariance' with a metric in an extended manifold; that makes non-relativistic models look similar to Lorentz-covariant relativistic models.

Galilean covariance therefore consists in enforcing Lorentz-like covariance in a $(4,1)$ space-time and then projecting the equations on a $(3,1)$ space-time, in such a way that the resulting theory is Galilean invariant [19-21]. We refer to the initial extended space-time as a Galilean manifold, which consists in the space of five-vectors

$$
x^{\mu}=\left(x^{1}, x^{2}, x^{3}, x^{4}, x^{5}\right)=(\mathbf{r}, t, s),
$$

whose coordinates transform under Galilean boosts as

$$
\begin{aligned}
\mathbf{r}^{\prime} & =\mathbf{r}-\mathbf{v} t, \\
t^{\prime} & =t, \\
s^{\prime} & =s-\mathbf{r} \cdot \mathbf{v}+\frac{1}{2} \mathbf{v}^{2} t .
\end{aligned}
$$

This transformation leaves invariant the scalar product

$$
(\mathbf{r}, t, s) \cdot\left(\mathbf{r}^{\prime}, t^{\prime}, s^{\prime}\right) \equiv \mathbf{r} \cdot \mathbf{r}^{\prime}-t s^{\prime}-t^{\prime} s,
$$


defined by the following metric,

$$
g^{\mu \nu}=\left(\begin{array}{ccccc}
1 & 0 & 0 & 0 & 0 \\
0 & 1 & 0 & 0 & 0 \\
0 & 0 & 1 & 0 & 0 \\
0 & 0 & 0 & 0 & -1 \\
0 & 0 & 0 & -1 & 0
\end{array}\right) .
$$

We shall refer to Eq. (1) as the Galilean metric, although it is in fact nothing but the Lorentz metric in $(4,1)$ space-time. The term "Galilean" indicates that an appropriate projection down to a $(3,1)$ space-time leads to a Galilean theory. Note that the extra parameter $s$ transforms like the phase of the quantum wave function that renders the Schrödinger equation invariant under Galilean transformations [19-21].

In this paper, we shall consider states $\Psi$ which satisfy

$$
\mathrm{i} \hbar \partial_{4} \Psi=E \Psi, \quad \mathrm{i} \hbar \partial_{5} \Psi=m \Psi,
$$

so that the five-momentum is

$$
p_{\mu}=-\mathrm{i} \hbar \partial_{\mu}=(\mathbf{p},-E,-m) .
$$

In short, the mass is $p^{4}=-p_{5}=m$, and the energy is $p^{5}=-p_{4}=E$. This provides us with an approach to non-relativistic theories that is rather systematical and similar to the relativistic Lorentz approach. Perhaps the best practical argument in favour of studying Galilean kinematics is the fact that there exist not one, but two Galilean limits of electromagnetism. This fact can be easily overlooked if one performs a hasty low-velocity approximation of relativistic equations [22].

This paper treats the non-relativistic counterpart of the relativistic Dirac potential, described in Ref. [23] for commutative and non-commutative spaces. The literature on noncommutative geometry is enormous and here we shall just cite a few papers investigating Galilei-invariant systems with non-commutative geometry in Refs. [24-29]. In order to compare our results with Ref. [23], we use a similar approach. Firstly, we modify the usual position and momentum operators,

$$
\left[r_{i}, r_{j}\right]=0, \quad\left[p_{i}, p_{j}\right]=0, \quad\left[r_{i}, p_{j}\right]=\mathrm{i} \hbar \delta_{i j},
$$

by applying a generalized 'Bopp shift' [30],

$$
\begin{aligned}
& \hat{r}_{i}=r_{i}-\frac{\Theta_{i j}}{2 \hbar} p_{j}=r_{i}+\frac{(\boldsymbol{\Theta} \times \mathbf{p})_{i}}{2 \hbar}, \\
& \hat{p}_{i}=p_{i}+\frac{\Omega_{i j}}{2 \hbar} r_{j}=p_{i}-\frac{(\boldsymbol{\Omega} \times \mathbf{r})_{i}}{2 \hbar} .
\end{aligned}
$$

Then the new operators $\hat{r}_{i}$ and $\hat{p}_{i}$ satisfy the following commutation relations:

$$
\left[\hat{r}_{i}, \hat{r}_{j}\right]=\mathrm{i} \Theta_{i j}, \quad\left[\hat{p}_{i}, \hat{p}_{j}\right]=\mathrm{i} \Omega_{i j}, \quad\left[\hat{r}_{i}, \hat{p}_{j}\right]=\mathrm{i} \hbar \Delta_{i j},
$$

where the matrix $\Delta_{i j}$ is given by

$$
\Delta_{i j}=\left(1+\frac{\boldsymbol{\Theta} \cdot \boldsymbol{\Omega}}{4 \hbar^{2}}\right) \delta_{i j}-\frac{\Omega_{i} \Theta_{j}}{4 \hbar^{2}},
$$

with $\Theta_{i j}=\epsilon_{i j k} \Theta_{k}, \Omega_{i j}=\epsilon_{i j k} \Omega_{k}$ (where $\Theta_{i}$ and $\Omega_{i}(i=1,2,3)$ are real parameters). 


\section{Galilean Dirac Oscillator in Commutative Space}

The Galilean version of the Dirac equation with gauge fields was first discussed by LévyLeblond in Ref. [31]; therefore it is sometimes called the "Lévy-Leblond equation". It can be obtained from the Galilean covariant formalism (see [32, 33] and Omote et al. [19-21]) by considering the Dirac-like equation,

$$
\gamma^{\mu} \partial_{\mu} \Psi=0, \quad \mu=1, \ldots, 5 .
$$

Although it looks like the massless Dirac equation, we mentioned in Eq. (2) that in our formalism, the mass of the particle is not zero and introduced via the extra $x^{5}$ coordinate. The mass will also appear in the non-minimal coupling (Eq. (11), below).

The gamma-matrices satisfy

$$
\left\{\gamma^{\mu}, \gamma^{\nu}\right\}=\gamma^{\mu} \gamma^{\nu}+\gamma^{v} \gamma^{\mu}=2 g^{\mu \nu},
$$

with the Galilean metric $g^{\mu \nu}$ given in Eq. (1). A four-dimensional representation is obtained when the Galilean Dirac matrices are taken to be

$$
\gamma^{i}=\left(\begin{array}{cc}
\sigma^{i} & 0 \\
0 & -\sigma^{i}
\end{array}\right), \quad \gamma^{4}=\left(\begin{array}{cc}
0 & 0 \\
-\sqrt{2} & 0
\end{array}\right), \quad \gamma^{5}=\left(\begin{array}{cc}
0 & \sqrt{2} \\
0 & 0
\end{array}\right),
$$

where each element is a two-by-two matrix, and with the Pauli spin matrices:

$$
\sigma^{1}=\left(\begin{array}{cc}
0 & 1 \\
1 & 0
\end{array}\right), \quad \sigma^{2}=\left(\begin{array}{cc}
0 & -\mathrm{i} \\
\mathrm{i} & 0
\end{array}\right), \quad \sigma^{3}=\left(\begin{array}{cc}
1 & 0 \\
0 & -1
\end{array}\right) .
$$

The adjoint spinor is given by

$$
\bar{\Psi}=\Psi^{\dagger} \zeta
$$

with

$$
\zeta=-\frac{i}{\sqrt{2}}\left(\gamma^{4}+\gamma^{5}\right)=\left(\begin{array}{cc}
0 & -i \\
\mathrm{i} & 0
\end{array}\right) .
$$

(This matrix satisfies the relation $\zeta \gamma^{\mu \dagger} \zeta=-\gamma^{\mu}$, and we observe that the bilinear expression $\bar{\Psi} \gamma^{\mu_{1}} \gamma^{\mu_{2}} \ldots \gamma^{\mu_{N}} \Psi$ is a rank- $N$ tensor under Galilean transformations.)

Thus Eqs. (2) and (7) lead to

$$
\gamma^{\mu} p_{\mu} \Psi=0
$$

and the Galilean Dirac oscillator is described by inserting the following non-minimal coupling (in a manner analogous to the Dirac oscillator described in Ref. [34, 35]):

$$
\mathbf{p} \rightarrow \mathbf{p}-\mathrm{i} m \omega \eta \mathbf{r} .
$$

We can choose the four-by-four matrix $\eta$ as

$$
\eta=\eta_{-} \eta_{+}=\left(\begin{array}{cc}
+1_{2 \times 2} & 0_{2 \times 2} \\
0_{2 \times 2} & -1_{2 \times 2}
\end{array}\right)
$$

where

$$
\eta_{ \pm}=\frac{i}{\sqrt{2}}\left(\gamma^{5} \pm \gamma^{4}\right)
$$

(Note that the matrix $\eta$ is not the same as in Ref. [36].) By substituting Eq. (11) into Eq. (10), the latter takes the form

$$
\left[\boldsymbol{\gamma} \cdot(\mathbf{p}-\mathrm{i} m \omega \eta \mathbf{r})+\gamma^{4} p_{4}+\gamma^{5} p_{5}\right] \Psi(x)=0 .
$$


If we express the four-spinor $\Psi$ in terms of two-spinors as follows,

$$
\Psi=\left(\begin{array}{l}
\varphi \\
\chi
\end{array}\right)
$$

then Eq. (13) leads to

$$
\begin{aligned}
& (\sigma \cdot \mathbf{p}-\mathrm{i} m \omega \sigma \cdot \mathbf{r}) \varphi+\sqrt{2} p_{5} \chi=0, \\
& (\sigma \cdot \mathbf{p}+\mathrm{i} m \omega \sigma \cdot \mathbf{r}) \chi+\sqrt{2} p_{4} \varphi=0 .
\end{aligned}
$$

If we multiply Eq. (15) by $(\sigma \cdot \mathbf{p}+\mathrm{i} m \omega \sigma \cdot \mathbf{r})$, and utilize

$$
\begin{aligned}
& (\sigma \cdot \mathbf{p})(\sigma \cdot \mathbf{p})=\mathbf{p}^{2}, \quad(\sigma \cdot \mathbf{r})(\sigma \cdot \mathbf{r})=\mathbf{r}^{2}, \\
& (\sigma \cdot \mathbf{r})(\sigma \cdot \mathbf{p})-(\sigma \cdot \mathbf{p})(\sigma \cdot \mathbf{r})=3 \mathrm{i} \hbar+2 \mathrm{i} \sigma \cdot(\mathbf{r} \times \mathbf{p}),
\end{aligned}
$$

we find

$$
\left(\mathbf{p}^{2}-3 m \hbar \omega-2 m \omega \sigma \cdot \mathbf{L}+m^{2} \omega^{2} \mathbf{r}^{2}\right) \varphi=-(\sigma \cdot \mathbf{p}+\mathrm{i} m \omega \sigma \cdot \mathbf{r}) \sqrt{2} p_{5} \chi .
$$

The right-hand side of this equation is similar to the first term of Eq. (16), so we multiply the previous equation by $\sqrt{2} p_{4}$ and obtain

$$
\left(\mathbf{p}^{2}-3 m \hbar \omega-2 m \omega \sigma \cdot \mathbf{L}+m^{2} \omega^{2} \mathbf{r}^{2}\right) \varphi=2 p_{4} p_{5} \varphi .
$$

If we replace $p_{4}=-E$ and $p_{5}=-m$, as in Eq. (2), and utilize $\mathbf{S} \equiv \frac{\hbar}{2} \sigma$, we obtain

$$
E \varphi=\left(\frac{\mathbf{p}^{2}}{2 m}+\frac{1}{2} m \omega^{2} \mathbf{r}^{2}-\frac{3}{2} \hbar \omega-\frac{2}{\hbar} \omega \mathbf{L} \cdot \mathbf{S}\right) \varphi .
$$

We proceed similarly with the other spinor component, $\chi$. First, we multiply Eq. (16) by $(\sigma \cdot \mathbf{p}-\mathrm{i} m \omega \sigma \cdot \mathbf{r})$, and obtain

$$
\left(\mathbf{p}^{2}+3 m \hbar \omega+2 m \omega \sigma \cdot \mathbf{L}+m^{2} \omega^{2} \mathbf{r}^{2}\right) \chi=-(\sigma \cdot \mathbf{p}+\mathrm{i} m \omega \sigma \cdot \mathbf{r}) \sqrt{2} p_{4} \varphi .
$$

The right-hand side of this equation is similar to the first term of Eq. (15); thus if we multiply the previous equation by $\sqrt{2} p_{5}$, we find

$$
E \chi=\left(\frac{\mathbf{p}^{2}}{2 m}+\frac{1}{2} m \omega^{2} \mathbf{r}^{2}+\frac{3}{2} \hbar \omega+\frac{2}{\hbar} \omega \mathbf{L} \cdot \mathbf{S}\right) \chi .
$$

Eqs. (17) and (18) are similar, except for the signs in their last two terms. Therefore, we shall hereafter combine them into a single equation as follows:

$$
E \psi_{s}=\left(\frac{\mathbf{p}^{2}}{2 m}+\frac{1}{2} m \omega^{2} \mathbf{r}^{2}+s \frac{3}{2} \hbar \omega+s \frac{2}{\hbar} \omega \mathbf{L} \cdot \mathbf{S}\right) \psi_{s},
$$

where $s=-1$ corresponds to $\psi_{s=-1}=\varphi$ and $s=+1$ corresponds to $\psi_{s=+1}=\chi$.

\section{Dirac Equation with Non-minimal Coupling in Non-commutative Phase Space}

In this section, we obtain the three-dimensional equation of motion and then we solve the two-dimensional equation. In Sect. 3.1, we develop the energy equation in three dimensions and consider general non-commutativity parameters $\boldsymbol{\Omega}$ and $\boldsymbol{\Theta}$. Then, in Sect. 3.2, we restrict ourselves to the two-dimensional space and solve the energy equation. 
3.1 General Non-commutativity Parameters in Three Dimensions

If we substitute Eqs. (4), (5) into Eq. (11) and then use Eq. (10), we find

$$
\begin{aligned}
& E \varphi=\frac{1}{2 m}(\sigma \cdot \hat{\mathbf{p}}+\mathrm{i} m \omega \sigma \cdot \hat{\mathbf{r}})(\sigma \cdot \hat{\mathbf{p}}-\mathrm{i} m \omega \sigma \cdot \hat{\mathbf{r}}) \varphi . \\
& E \chi=\frac{1}{2 m}(\sigma \cdot \hat{\mathbf{p}}-\mathrm{i} m \omega \sigma \cdot \hat{\mathbf{r}})(\sigma \cdot \hat{\mathbf{p}}+\mathrm{i} m \omega \sigma \cdot \hat{\mathbf{r}}) \chi .
\end{aligned}
$$

These equations can be simplified by observing that (see details in appendix)

$$
\begin{aligned}
& (\sigma \cdot \hat{\mathbf{p}})(\sigma \cdot \hat{\mathbf{p}})=-\frac{2}{\hbar} \mathbf{S} \cdot \boldsymbol{\Omega}+\mathbf{p}^{2}-\frac{1}{\hbar} \boldsymbol{\Omega} \cdot \mathbf{L}+\frac{1}{4 \hbar^{2}}(\boldsymbol{\Omega} \times \mathbf{r})^{2}, \\
& (\sigma \cdot \hat{\mathbf{r}})(\sigma \cdot \hat{\mathbf{r}})=-\frac{2}{\hbar} \mathbf{S} \cdot \Theta+\mathbf{r}^{2}-\frac{1}{\hbar} \Theta \cdot \mathbf{L}+\frac{1}{4 \hbar^{2}}(\Theta \times \mathbf{p})^{2},
\end{aligned}
$$

as well as (see Appendix for the proof)

$$
\begin{aligned}
(\sigma \cdot \hat{\mathbf{r}})(\sigma \cdot \hat{\mathbf{p}})-(\sigma \cdot \hat{\mathbf{p}})(\sigma \cdot \hat{\mathbf{r}})= & \frac{4 \mathrm{i}}{\hbar} \mathbf{S} \cdot \mathbf{L}+3 \mathrm{i} \hbar-\frac{2 \mathrm{i}}{\hbar^{2}}(\mathbf{S} \times \mathbf{r}) \cdot(\boldsymbol{\Omega} \times \mathbf{r}) \\
& +\frac{2 \mathrm{i}}{\hbar^{2}}(\mathbf{S} \times \mathbf{p}) \cdot(\boldsymbol{\Theta} \times \mathbf{p})+\frac{\mathrm{i}}{\hbar^{3}}[(\mathbf{S} \cdot \boldsymbol{\Omega})(\boldsymbol{\Theta} \cdot \mathbf{L}) \\
& +[\boldsymbol{\Theta} \cdot(\mathbf{p} \times \boldsymbol{\Omega})](\mathbf{S} \cdot \mathbf{r})] \\
& +\mathrm{i} \frac{i \sigma \cdot(\boldsymbol{\Omega} \times \boldsymbol{\Theta})+2 \boldsymbol{\Theta} \cdot \boldsymbol{\Omega}}{4 \hbar}
\end{aligned}
$$

so that we find that the energy equation takes the form

$$
\begin{aligned}
E \psi_{s}= & {\left[\frac{\mathbf{p}^{2}}{2 m}+\frac{1}{2} m \omega^{2} \mathbf{r}^{2}+\frac{1}{8 m \hbar^{2}}\left((\mathbf{r} \times \boldsymbol{\Omega})^{2}+m^{2} \omega^{2}(\mathbf{p} \times \boldsymbol{\Theta})^{2}\right)\right.} \\
& -\frac{1}{2 m \hbar}\left(\boldsymbol{\Omega}+m^{2} \omega^{2} \boldsymbol{\Theta}\right) \cdot(\mathbf{L}+2 \mathbf{S}) \\
& +s \omega\left(\frac{2}{\hbar} \mathbf{L} \cdot \mathbf{S}+\frac{3}{2} \hbar-\frac{2}{\hbar^{2}}(\mathbf{S} \times \mathbf{r}) \cdot(\boldsymbol{\Omega} \times \mathbf{r})+\frac{2}{\hbar^{2}}(\mathbf{S} \times \mathbf{p}) \cdot(\boldsymbol{\Theta} \times \mathbf{p})\right. \\
& +\frac{1}{2 \hbar^{3}}[(\mathbf{S} \cdot \boldsymbol{\Omega})(\boldsymbol{\Theta} \cdot \mathbf{L})+[\boldsymbol{\Theta} \cdot(\mathbf{p} \times \boldsymbol{\Omega})](\mathbf{S} \cdot \mathbf{r})] \\
& \left.\left.+\frac{\mathrm{i} \sigma \cdot(\boldsymbol{\Omega} \times \boldsymbol{\Theta})+2 \boldsymbol{\Theta} \cdot \boldsymbol{\Omega}}{8 \hbar}\right)\right] \psi_{s} .
\end{aligned}
$$

If we denote by $\alpha_{r \Omega}\left(\right.$ resp. $\left.\alpha_{p \Theta}\right)$ the angle between $\mathbf{r}$ and $\boldsymbol{\Omega}$ (resp. $\mathbf{p}$ and $\boldsymbol{\Theta}$ ), we see that Eq. (25) can be rewritten as

$$
\begin{aligned}
E \psi_{s}= & {\left[\frac{1}{2 m}\left(1+\frac{m^{2} \omega^{2} \boldsymbol{\Theta}^{2} \sin ^{2} \alpha_{p \Theta}}{4 \hbar^{2}}\right) \mathbf{p}^{2}+\frac{1}{2} m \omega^{2}\left(1+\frac{\boldsymbol{\Omega}^{2} \sin ^{2} \alpha_{r \Omega}}{4 m^{2} \omega^{2} \hbar^{2}}\right) \mathbf{r}^{2}+s \frac{3}{2} \hbar \omega\right.} \\
& +s \frac{2}{\hbar} \omega \mathbf{L} \cdot \mathbf{S}-\frac{1}{2 m \hbar}\left(\boldsymbol{\Omega}+m^{2} \omega^{2} \boldsymbol{\Theta}\right) \cdot \mathbf{L}+\frac{1}{2 \hbar^{3}}(\mathbf{S} \cdot \boldsymbol{\Omega})(\boldsymbol{\Theta} \cdot \mathbf{L}) \\
& -\frac{1}{m \hbar}\left(\boldsymbol{\Omega}+m^{2} \omega^{2} \boldsymbol{\Theta}\right) \cdot \mathbf{S}+s \omega\left(\frac{2}{\hbar^{2}}(\mathbf{S} \times \mathbf{p}) \cdot(\boldsymbol{\Theta} \times \mathbf{p})-\frac{2}{\hbar^{2}}(\mathbf{S} \times \mathbf{r}) \cdot(\boldsymbol{\Omega} \times \mathbf{r})\right. \\
& \left.\left.+\frac{1}{2 \hbar^{3}} \boldsymbol{\Theta} \cdot(\mathbf{p} \times \boldsymbol{\Omega})(\mathbf{S} \cdot \mathbf{r})+\frac{\mathrm{i} \sigma \cdot(\boldsymbol{\Omega} \times \boldsymbol{\Theta})+2 \boldsymbol{\Theta} \cdot \boldsymbol{\Omega}}{8 \hbar}\right)\right] \psi_{s} .
\end{aligned}
$$


We can express it in a slightly more compact form by defining

$$
\begin{gathered}
\tilde{M}=m\left(1+\frac{m^{2} \omega^{2} \boldsymbol{\Theta}^{2} \sin ^{2} \alpha_{p \Theta}}{4 \hbar^{2}}\right)^{-1}, \quad \tilde{M} \tilde{\Xi}^{2}=m \omega^{2}\left(1+\frac{\boldsymbol{\Omega}^{2} \sin ^{2} \alpha_{r \Omega}}{4 m^{2} \omega^{2} \hbar^{2}}\right), \\
\mathbf{D}(\boldsymbol{\Theta}, \boldsymbol{\Omega}, m, \omega, s)=s \frac{2}{\hbar} \omega \mathbf{S}-\frac{1}{2 m \hbar}\left(\boldsymbol{\Omega}+m^{2} \omega^{2} \boldsymbol{\Theta}\right)+\frac{1}{2 \hbar^{3}}(\mathbf{S} \cdot \boldsymbol{\Omega}) \boldsymbol{\Theta}
\end{gathered}
$$

Then we can rewrite Eq. (26) as

$$
\begin{aligned}
E \psi_{s}= & {\left[\frac{1}{2 \tilde{M}} \mathbf{p}^{2}+\frac{1}{2} \tilde{M} \tilde{\Xi}^{2} \mathbf{r}^{2}+\mathbf{D} \cdot \mathbf{L}+s \frac{3}{2} \hbar \omega\right.} \\
& -\frac{1}{m \hbar}\left(\boldsymbol{\Omega}+m^{2} \omega^{2} \boldsymbol{\Theta}\right) \cdot \mathbf{S}+s \omega\left(\frac{2}{\hbar^{2}}(\mathbf{S} \times \mathbf{p}) \cdot(\boldsymbol{\Theta} \times \mathbf{p})-\frac{2}{\hbar^{2}}(\mathbf{S} \times \mathbf{r}) \cdot(\boldsymbol{\Omega} \times \mathbf{r})\right. \\
& \left.\left.+\frac{1}{2 \hbar^{3}} \boldsymbol{\Theta} \cdot(\mathbf{p} \times \boldsymbol{\Omega})(\mathbf{S} \cdot \mathbf{r})+\frac{\mathrm{i} \sigma \cdot(\boldsymbol{\Omega} \times \boldsymbol{\Theta})+2 \boldsymbol{\Theta} \cdot \boldsymbol{\Omega}}{8 \hbar}\right)\right] \psi_{s}
\end{aligned}
$$

In the next section, we shall find the energy eigenvalues for the two-dimensional version of this equation. Thus many terms will become constant, thereby rendering the calculations more manageable.

\subsection{Energy Spectrum in Two Dimensions}

Hereafter, we confine ourselves to planar systems in order to compare with the analogous relativistic investigation of Ref. [23]. Therefore the space is described by coordinates $(x, y)$ with the corresponding momentum components $\left(p_{x}, p_{y}\right)$. If we choose these components as non-commuting in Eq. (6), this amounts to taking the following parameters:

$$
\boldsymbol{\Theta}=(0,0, \Theta), \quad \boldsymbol{\Omega}=(0,0, \Omega), \quad \mathbf{S}=\left(0,0, S_{z}\right) .
$$

Then Eq. (29) becomes

$$
\begin{aligned}
E \psi_{s}= & {\left[\frac{1}{2 \tilde{M}}\left(p_{x}^{2}+p_{y}^{2}\right)+\frac{1}{2} \tilde{M} \tilde{\Xi}^{2}\left(x^{2}+y^{2}\right)+D_{z} L_{z}+s \frac{3}{2} \hbar \omega-\frac{1}{m \hbar}\left(\Omega+m^{2} \omega^{2} \Theta\right) S_{z}+\right.} \\
& \left.+s \omega\left(\frac{2 S_{z} \Theta}{\hbar^{2}}\left(p_{x}^{2}+p_{y}^{2}\right)-\frac{2 S_{z} \Omega}{\hbar^{2}}\left(x^{2}+y^{2}\right)+\frac{\Theta \Omega}{4 \hbar}\right)\right] \psi_{s},
\end{aligned}
$$

or

$$
\begin{aligned}
E \psi_{s}= & {\left[\left(\frac{1}{2 \tilde{M}}+\frac{2 s \omega S_{z} \Theta}{\hbar^{2}}\right)\left(p_{x}^{2}+p_{y}^{2}\right)+\left(\frac{1}{2} \tilde{M} \tilde{\Xi}^{2}-\frac{2 s \omega S_{z} \Omega}{\hbar^{2}}\right)\left(x^{2}+y^{2}\right)+D_{z} L_{z}\right.} \\
& \left.+s \omega\left(\frac{3}{2} \hbar+\frac{\Theta \Omega}{4 \hbar}\right)-\frac{1}{m \hbar}\left(\Omega+m^{2} \omega^{2} \Theta\right) S_{z}\right] \psi_{s}
\end{aligned}
$$

If we take $\psi_{s}$ as an eigenvector of $S_{z}$, and use the matrix representation for $\sigma^{3}$ we find

$$
\psi_{s}=\left(\begin{array}{c}
\psi_{s}^{+} \\
\psi_{s}^{-}
\end{array}\right) \rightarrow S_{z} \psi_{s}=\frac{\hbar}{2}\left(\begin{array}{c}
\psi_{s}^{+} \\
-\psi_{s}^{-}
\end{array}\right) .
$$

Henceforth we will use the notation $\psi_{s} \rightarrow \psi_{s}^{s_{z}}$ so that $S_{z} \psi_{s}=\frac{\hbar}{2} s_{z} \psi_{s}, s_{z}= \pm 1$. It follows

$$
\begin{aligned}
E \psi_{s}^{s_{z}}= & {\left[\left(\frac{1}{2 \tilde{M}}+\frac{s s_{z} \omega \Theta}{\hbar}\right)\left(p_{x}^{2}+p_{y}^{2}\right)+\left(\frac{1}{2} \tilde{M} \tilde{\Xi}^{2}-\frac{s s_{z} \omega \Omega}{\hbar}\right)\left(x^{2}+y^{2}\right)+d_{z} L_{z}\right.} \\
& \left.+s \omega\left(\frac{3}{2} \hbar+\frac{\Theta \Omega}{4 \hbar}\right)-\frac{1}{2 m}\left(\Omega+m^{2} \omega^{2} \Theta\right) s_{z}\right] \psi_{s},
\end{aligned}
$$


where

$$
d_{z}=s s_{z} \omega-\frac{1}{2 m \hbar}\left(\Omega+m^{2} \omega^{2} \Theta\right)+\frac{1}{4 \hbar^{2}} s_{z} \Omega \Theta .
$$

If we absorb the energy into

$$
\mathcal{E}(s, \omega)=E-s \omega\left(\frac{3}{2} \hbar+\frac{\Theta \Omega}{4 \hbar}\right)+\frac{1}{2 m}\left(\Omega+m^{2} \omega^{2} \Theta\right) s_{z},
$$

and define the following

$$
\frac{1}{2 M}=\frac{1}{2 \tilde{M}}+\frac{s s_{z} \omega \Theta}{\hbar}, \quad \frac{1}{2} M \Xi^{2}=\frac{1}{2} \tilde{M} \tilde{\Xi}^{2}-\frac{s s_{z} \omega \Omega}{\hbar},
$$

then Eq. (31) takes the simpler form

$$
\mathcal{E} \psi_{s}^{s_{z}}=\left[\frac{1}{2 M}\left(p_{x}^{2}+p_{y}^{2}\right)+\frac{1}{2} M \Xi^{2}\left(x^{2}+y^{2}\right)+d_{z} L_{z}\right] \psi_{s}^{s_{z}} .
$$

Notice that depending on the values of $s, s_{z}, \Theta$ and $\Omega$, the parameters $M$ and $\Xi^{2}$ can be singular so that there may be a region on the parameters space where the equation will not be valid.

From this point on, let us work with polar coordinates $(r, \theta)$, so that Eq. (35) becomes

$$
\mathcal{E} \psi_{s}^{s_{z}}=\left[-\frac{\hbar^{2}}{2 M}\left(\frac{1}{r} \frac{\partial}{\partial r}+\frac{\partial^{2}}{\partial r^{2}}+\frac{1}{r^{2}} \frac{\partial^{2}}{\partial \theta^{2}}\right)+\frac{1}{2} M \Xi^{2} r^{2}+d_{z}\left(-\mathrm{i} \hbar \frac{\partial}{\partial \theta}\right)\right] \psi_{s}^{s_{z}} .
$$

Note also, for later use, that $\tilde{M}$ and $\tilde{\Xi}$ in Eq. (27) reduce to

$$
\tilde{M}=m\left(1+\frac{m^{2} \omega^{2} \Theta^{2}}{4 \hbar^{2}}\right)^{-1}, \quad \tilde{\Xi}^{2}=\omega^{2}\left(1+\frac{\Omega^{2}}{4 m^{2} \omega^{2} \hbar^{2}}\right)\left(1+\frac{m^{2} \omega^{2} \Theta^{2}}{4 \hbar^{2}}\right),
$$

and that

$$
\tilde{M} \tilde{\Xi}^{2}=m \omega^{2}\left(1+\frac{\Omega^{2}}{4 m^{2} \omega^{2} \hbar^{2}}\right) .
$$

In order to solve Eq. (36), we separate the variables as follows,

$$
\psi_{s}^{s_{z}}(r, \theta)=\chi_{s}^{s_{z}}(r) \exp \left(\mathrm{im}_{\theta} \theta\right), \quad m_{\theta}=0, \pm 1, \pm 2, \ldots
$$

so that Eq. (36) leads to

$$
\left[\frac{\hbar^{2}}{2 M}\left(\frac{1}{r} \frac{d}{d r}+\frac{d^{2}}{d r^{2}}-\frac{m_{\theta}^{2}}{r^{2}}\right)-\frac{1}{2} M \Xi^{2} r^{2}+\overline{\mathcal{E}}\right] \chi_{s}^{s_{z}}(r)=0,
$$

where we have defined

$$
\overline{\mathcal{E}}=\mathcal{E}-\hbar m_{\theta} d_{z}
$$

We determine the energy spectrum by solving Eq. (40). If we divide each term of Eq. (40) by $\hbar \Xi$, then the operator becomes dimensionless:

$$
\left[\frac{\hbar}{2 M \Xi}\left(\frac{1}{r} \frac{d}{d r}+\frac{d^{2}}{d r^{2}}-\frac{m_{\theta}^{2}}{r^{2}}\right)-\frac{1}{2} \frac{M \Xi}{\hbar} r^{2}+\frac{\overline{\mathcal{E}}}{\hbar \Xi}\right] \chi_{s}^{s_{z}}=0 .
$$

If we replace the variable $r$ by

$$
y \equiv \frac{1}{2} \frac{M \Xi}{\hbar} r^{2}
$$


then we obtain the linear homogeneous second-order differential equation:

$$
y \frac{d^{2} u(y)}{d y^{2}}+\frac{d u(y)}{d y}+\left(\frac{\overline{\mathcal{E}}}{\hbar \Xi}-y-\frac{m_{\theta}^{2}}{4 y}\right) u(y)=0 .
$$

This has the same form as Eq. (32) of our previous article on the spin-zero DKP oscillator [1]. Accordingly, let us introduce the function $f(y)$, related to $u(y)$ through

$$
u(y)=\exp (-y) y^{\left|m_{\theta}\right| / 2} f(y)
$$

so that, when substituted into Eq. (43), it leads to

$$
y \frac{d^{2} f(y)}{d y^{2}}+\left(\left|m_{\theta}\right|+1-2 y\right) \frac{d f(y)}{d y}+\left(\frac{\overline{\mathcal{E}}}{\hbar \Xi}-\left|m_{\theta}\right|-1\right) f(y)=0 .
$$

If we define

$$
w \equiv 2 y, \quad \gamma \equiv\left|m_{\theta}\right|+1, \quad 2 \alpha \equiv\left|m_{\theta}\right|+1-\frac{\overline{\mathcal{E}}}{\hbar \Xi},
$$

then we obtain the Kummer's differential equation,

$$
w \frac{d^{2} f(w)}{d w^{2}}+(\gamma-w) \frac{d f(w)}{d w}-\alpha f(w)=0 .
$$

As the spinless DKP oscillator [1], its solution is the confluent hypergeometric function (see Sect. 13.1.1 of Ref. [37]),

$$
f(w)=A\left[{ }_{1} F_{1}(\alpha ; \gamma ; w)\right],
$$

( $A$ is a normalization constant) where

$$
{ }_{1} F_{1}(\alpha ; \gamma ; w)=1+\frac{\alpha w}{\gamma}+\frac{(\alpha)_{2} w^{2}}{(\gamma)_{2} 2 !}+\cdots+\frac{(\alpha)_{n} w^{n}}{(\gamma)_{n} n !}+\cdots,
$$

with the Pocchammer symbol $(\cdots)_{n}$ :

$$
(a)_{n} \equiv a(a+1)(a+2) \cdots(a+n-1), \quad(a)_{0} \equiv 1 .
$$

The solution can also be expressed in terms of associated Laguerre polynomials.

In order to express the general solutions of the wave function in terms of the initial polar variables, $r$ and $\theta$, we utilize Eqs. (39), (42), (44), (45), and (46) and find

$$
\begin{aligned}
\psi_{s}^{s_{z}}(r, \theta)= & N_{s}^{s_{z}} \exp \left(-\frac{M \Xi r^{2}}{2 \hbar}+\mathrm{i} m_{\theta} \theta\right) r^{\left|m_{\theta}\right|} \\
& \times{ }_{1} F_{1}\left(\frac{1}{2}\left(\left|m_{\theta}\right|+1-\frac{\mathcal{E}-\hbar m_{\theta} d_{z}}{\hbar \Xi}\right) ;\left|m_{\theta}\right|+1 ; \frac{M \Xi}{\hbar} r^{2}\right) .
\end{aligned}
$$

The constant $A$ in Eq. (46) has been absorbed within the normalization constant spinor $N_{s}^{s_{z}}$, along with other constants. Note that $s$, which labels the two-spinors, appears in $d_{z}$ via Eq. (28). Let us also remind that the variable $\Xi$ is in Eq. (37), $\mathcal{E}$ is in Eq. (33), and $M \Xi$ is given in Eq. (38).

From the boundary condition $w \rightarrow \infty$ (or $r \rightarrow \infty)$, at which $f(w) \rightarrow 0$ (so that $\psi_{s} \rightarrow 0$ ), we find

$$
\alpha=\frac{1}{2}\left(\left|m_{\theta}\right|+1-\frac{\overline{\mathcal{E}}}{\hbar \Xi}\right)=-n, \quad n=0,1,2, \ldots
$$

From Eqs. (41) and (48), we find

$$
\mathcal{E}=\left(2 n+\left|m_{\theta}\right|+1\right) \hbar \Xi+m_{\theta} \hbar d_{z} .
$$


Therefore, the energy eigenvalue, $E_{n, m_{\theta}, s, s_{z}}$, of the two-dimensional DKP oscillator is obtained from Eq. (33) in which we substitute Eq. (49). This gives

$$
E_{n, m_{\theta}, s, s_{z}}=\left(2 n+\left|m_{\theta}\right|+1\right) \hbar \Xi+\hbar m_{\theta} d_{z}+s \omega\left(\frac{3}{2} \hbar+\frac{\Theta \Omega}{4 \hbar}\right)-\frac{1}{2 m} s_{z}\left(\Omega+m^{2} \omega^{2} \Theta\right)
$$

where $d_{z}$ is given in Eq. (32), and $\Xi$ is obtained from Eqs. (34) and (37). If we multiply together the two terms in Eq. (34), they give

$$
\frac{1}{4} \Xi^{2}=\left(\frac{1}{2} \tilde{M} \tilde{\Xi}^{2}-\frac{2 s \omega S_{z} \Omega}{\hbar^{2}}\right)\left(\frac{1}{2 \tilde{M}}+\frac{2 s \omega S_{z} \Theta}{\hbar^{2}}\right) .
$$

Then we substitute $\tilde{M}$ and $\tilde{\Xi}$ from Eqs. (37) and (38), and obtain

$$
\Xi^{2}=4\left[\frac{1}{2} m \omega^{2}\left(1+\frac{\Omega^{2}}{4 m^{2} \omega^{2} \hbar^{2}}\right)-\frac{s s_{z} \omega \Omega}{\hbar}\right]\left[\frac{1}{2 m}\left(1+\frac{m^{2} \omega^{2} \Theta^{2}}{4 \hbar^{2}}\right)+\frac{s s_{z} \omega \Theta}{\hbar}\right] .
$$

To summarize, the energy eigenvalues are

$$
\begin{aligned}
E_{n, m_{\theta}, s, s_{z}}= & 2\left(2 n+\left|m_{\theta}\right|+1\right) \hbar \sqrt{\frac{1}{2} m \omega^{2}\left(1+\frac{\Omega^{2}}{4 m^{2} \omega^{2} \hbar^{2}}\right)-\frac{s s_{z} \omega \Omega}{\hbar}} \\
& \times \sqrt{\frac{1}{2 m}\left(1+\frac{m^{2} \omega^{2} \Theta^{2}}{4 \hbar^{2}}\right)+\frac{s s_{z} \omega \Theta}{\hbar}} \\
& +\hbar m_{\theta}\left(s s_{z} \omega-\frac{1}{2 m \hbar}\left(\Omega+m^{2} \omega^{2} \Theta\right)+\frac{s_{z} \Omega \Theta}{4 \hbar^{2}}\right) \\
& +s \omega\left(\frac{3}{2} \hbar+\frac{\Theta \Omega}{4 \hbar}\right)-\frac{s_{z}}{2 m}\left(\Omega+m^{2} \omega^{2} \Theta\right) .
\end{aligned}
$$

In the commutative limit, $\Omega=0, \Theta=0$, Eq. (50) reduces to

$$
E_{n, m_{\theta}, s, s_{z}}=\left(2 n+\left|m_{\theta}\right|+1\right) \hbar \omega+s s_{z} m_{\theta} \hbar \omega+\frac{3}{2} s \hbar \omega .
$$

\section{Conserved Currents and Normalization of the Wave Function}

The purpose of this section is to determine the normalization constant of the wave function in the previous section. First, we determine the conserved current in the commutative space, with and without non-minimal coupling. Then, we repeat the calculation in the noncommutative space, and thereby determine the constant.

\subsection{Conserved Current in Commutative Space}

First let us consider a commutative space, for which the non-coupled field equations are

$$
\gamma^{\mu} \partial_{\mu} \Psi=0, \quad \bar{\Psi} \gamma^{\mu} \overleftarrow{\partial_{\mu}}=0
$$

When the first equation is contracted with $\bar{\Psi}$ (defined in Eqs. (8) and (9)) from the left, and the second with $\Psi$ from the right, the sum of the two equations give

$$
\bar{\Psi} \gamma^{\mu} \partial_{\mu} \Psi+\partial_{\mu} \bar{\Psi} \gamma^{\mu} \Psi=\partial_{\mu}\left(\bar{\Psi} \gamma^{\mu} \Psi\right)=0
$$

showing that

$$
J^{\mu} \equiv \bar{\psi} \gamma^{\mu} \psi
$$


is a conserved current.

Next, consider the Dirac-like equation in a commutative space, with the non-minimal coupling from Eq. (11). In the coordinate space, Eq. (13) takes the form

$$
\left(\hbar \gamma^{\mu} \partial_{\mu}+m \omega \gamma^{\mu} \delta_{\mu}^{i} \eta r_{i}\right) \Psi=0,
$$

with $\eta$ defined in Eq. (12). The complex conjugate transpose of this equation leads to

$$
\begin{aligned}
0 & =\left[\left(\hbar \gamma^{\mu} \partial_{\mu}+m \omega \gamma^{\mu} \delta_{\mu}^{i} \eta r_{i}\right) \Psi\right]^{\dagger} \\
& =\Psi^{\dagger}\left(\hbar \gamma^{\dagger \mu} \overleftarrow{\partial_{\mu}}+m \omega \delta_{\mu}^{i} \eta^{\dagger} \gamma^{\dagger \mu} r_{i}\right) \\
& =\bar{\Psi} \zeta\left(\hbar \gamma^{\dagger \mu} \overleftarrow{\partial_{\mu}}+m \omega \delta_{\mu}^{i} \eta^{\dagger} \gamma^{\dagger \mu} r_{i}\right) \\
& =\bar{\Psi}\left(\hbar \gamma^{\mu} \zeta \overleftarrow{\partial_{\mu}}+m \omega \delta_{\mu}^{i} \zeta \eta^{\dagger} \gamma^{\dagger \mu} r_{i}\right)
\end{aligned}
$$

And by using Eqs. (9) and (12), we find

$$
\begin{aligned}
\zeta \eta^{\dagger} & =\left(\begin{array}{cc}
0 & -\mathrm{i} \\
\mathrm{i} & 0
\end{array}\right)\left(\begin{array}{cc}
1_{2 \times 2} & 0 \\
0 & -1_{2 \times 2}
\end{array}\right)=\left(\begin{array}{ll}
0 & \mathrm{i} \\
\mathrm{i} & 0
\end{array}\right) \\
& =-\left(\begin{array}{cc}
1_{2 \times 2} & 0 \\
0 & -1_{2 \times 2}
\end{array}\right)\left(\begin{array}{cc}
0 & -\mathrm{i} \\
\mathrm{i} & 0
\end{array}\right)=-\eta \zeta,
\end{aligned}
$$

so that

$$
\zeta \eta^{\dagger} \gamma^{\dagger \mu}=-\eta \zeta \gamma^{\dagger \mu}=\eta \gamma^{\mu} \zeta
$$

and we obtain

$$
0=\bar{\Psi}\left(\hbar \gamma^{\mu} \zeta \overleftarrow{\partial_{\mu}}+m \omega \delta_{\mu}^{i} \eta \gamma^{\mu} \zeta r_{i}\right)
$$

If we multiply this expression by $\zeta$ from the right, we obtain $0=\bar{\Psi}\left(\hbar \gamma^{\mu} \overleftarrow{\partial_{\mu}}+m \omega \delta_{\mu}^{i} \eta \gamma^{\mu} r_{i}\right)$ And by taking into account that $\eta \gamma^{\mu}=-\gamma^{\mu} \eta$, we find $\bar{\Psi}\left(\hbar \gamma^{\mu} \overleftarrow{\partial_{\mu}}-m \omega \delta_{\mu}^{i} \gamma^{\mu} \eta r_{i}\right)=0$

To sum up, we have the following two equations:

$$
\begin{aligned}
& \left(\hbar \gamma^{\mu} \partial_{\mu}+m \omega \gamma^{\mu} \delta_{\mu}^{i} \eta r_{i}\right) \Psi=0 \\
& \bar{\Psi}\left(\hbar \gamma^{\mu} \overleftarrow{\partial_{\mu}}-m \omega \delta_{\mu}^{i} \gamma^{\mu} \eta r_{i}\right)=0 .
\end{aligned}
$$

As we did in the free case, we multiply the first equation by $\bar{\Psi}$ from the left, and the second with $\Psi$ from the right. The sum of the resulting equations gives

$$
\hbar \bar{\Psi} \gamma^{\mu} \partial_{\mu} \Psi+\hbar \partial_{\mu} \bar{\Psi} \gamma^{\mu} \Psi=\hbar \partial_{\mu}\left(\bar{\Psi} \gamma^{\mu} \Psi\right)=0 .
$$

This shows that, in the commutative space, the conserved current is given by Eq. (52) with and without minimal coupling.

\subsection{Conserved Current with Non-minimal Coupling in Non-commutative Space}

In the non-commutative space the field equation with non-minimal coupling becomes

$$
\left(\hbar \gamma^{\mu} \hat{\partial}_{\mu}+m \omega \gamma^{\mu} \delta_{\mu}^{i} \eta \hat{r}_{i}\right) \Psi=0 .
$$

Where we use Eqs. (4) and (5). Note that Eq. (5) leads to

$$
\hbar \hat{\partial}_{i}=\hbar \partial_{i}-\frac{\mathrm{i}(\boldsymbol{\Omega} \times \mathbf{r})_{i}}{2 \hbar}
$$


so that the previous equation becomes

$$
\left(\hbar \gamma^{\mu} \partial_{\mu}-\mathrm{i} \gamma^{\mu} \delta_{\mu}^{i} \frac{(\boldsymbol{\Omega} \times \mathbf{r})_{i}}{2 \hbar}+m \omega \gamma^{\mu} \delta_{\mu}^{i} \eta r_{i}+m \omega \gamma^{\mu} \delta_{\mu}^{i} \eta \frac{(\boldsymbol{\Theta} \times \mathbf{p})_{i}}{2 \hbar}\right) \Psi=0 .
$$

The adjoint equation then reads

$$
\begin{aligned}
0 & =\left[\left(\hbar \gamma^{\mu} \partial_{\mu}+\mathrm{i} \gamma^{\mu} \delta_{\mu}^{i} \frac{(\boldsymbol{\Omega} \times \mathbf{r})_{i}}{2 \hbar}+m \omega \gamma^{\mu} \delta_{\mu}^{i} \eta r_{i}+m \omega \gamma^{\mu} \delta_{\mu}^{i} \eta \frac{(\boldsymbol{\Theta} \times \mathbf{p})_{i}}{2 \hbar}\right) \Psi\right]^{\dagger} \zeta \\
& =\Psi^{\dagger}\left(\hbar \gamma^{\mu} \zeta \overleftarrow{\partial_{\mu}}+\mathrm{i} \gamma^{\mu} \zeta \delta_{\mu}^{i} \frac{(\boldsymbol{\Omega} \times \mathbf{r})_{i}}{2 \hbar}+m \omega \delta_{\mu}^{i} \eta \gamma^{\mu} \zeta r_{i}+m \omega \zeta \eta^{\dagger} \gamma^{\mu \dagger} \delta_{\mu}^{i} \frac{(-\mathrm{i} \hbar \boldsymbol{\Theta} \times \overleftarrow{\nabla})_{i}}{2 \hbar}\right) \zeta \\
& =\bar{\Psi}\left(\hbar \gamma^{\mu} \overleftarrow{\partial_{\mu}}+\mathrm{i} \gamma^{\mu} \delta_{\mu}^{i} \frac{(\boldsymbol{\Omega} \times \mathbf{r})_{i}}{2 \hbar}-m \omega \delta_{\mu}^{i} \gamma^{\mu} \eta r_{i}+m \omega \gamma^{\mu} \eta \delta_{\mu}^{i} \frac{(\boldsymbol{\Theta} \times \overleftarrow{\mathbf{p}})_{i}}{2 \hbar}\right)
\end{aligned}
$$

where we utilized the following result:

$$
\begin{aligned}
{\left[m \omega \gamma^{\mu} \delta_{\mu}^{i} \eta \frac{(\boldsymbol{\Theta} \times \mathbf{p})_{i}}{2 \hbar} \Psi\right]^{\dagger} } & =m \omega \psi^{\dagger} \eta^{\dagger} \gamma^{\dagger \mu} \delta_{\mu}^{i} \overleftarrow{\left[\frac{(\boldsymbol{\Theta} \times \mathbf{p})_{i}}{2 \hbar}\right]^{\dagger}} \\
& =m \omega \psi^{\dagger} \eta^{\dagger} \gamma^{\dagger \mu} \delta_{\mu}^{i} \overleftarrow{\left[\frac{(\boldsymbol{\Theta} \times i \boldsymbol{\nabla})_{i}}{2 \hbar}\right]^{\dagger}} \\
& =m \omega \psi^{\dagger} \eta^{\dagger} \gamma^{\dagger \mu} \delta_{\mu}^{i}\left[\frac{(-i \boldsymbol{\Theta} \times \overleftarrow{\nabla})_{i}}{2 \hbar}\right]
\end{aligned}
$$

If we proceed as for the commutative case, that is, by taking the direct and adjoint equations properly contracted with $\bar{\Psi}$ and $\Psi$, leads to

$$
\begin{gathered}
\bar{\Psi}\left(\hbar \gamma^{\mu} \overleftarrow{\partial}_{\mu}+\mathrm{i} \gamma^{\mu} \delta_{\mu}^{i} \frac{(\boldsymbol{\Omega} \times \mathbf{r})_{i}}{2 \hbar}-m \omega \delta_{\mu}^{i} \gamma^{\mu} \eta r_{i}+m \omega \gamma^{\mu} \eta \delta_{\mu}^{i} \frac{(\boldsymbol{\Theta} \times \overleftarrow{\mathbf{p}})_{i}}{2 \hbar}\right) \Psi=0 \\
\bar{\Psi}\left(\hbar \gamma^{\mu} \partial_{\mu}-\mathrm{i} \gamma^{\mu} \delta_{\mu}^{i} \frac{(\boldsymbol{\Omega} \times \mathbf{r})_{i}}{2 \hbar}+m \omega \gamma^{\mu} \delta_{\mu}^{i} \eta r_{i}+m \omega \gamma^{\mu} \delta_{\mu}^{i} \eta \frac{(\boldsymbol{\Theta} \times \mathbf{p})_{i}}{2 \hbar}\right) \Psi=0
\end{gathered}
$$

and the sum of these terms is equal to

$$
\hbar\left(\partial_{\mu} \bar{\Psi}\right) \gamma^{\mu} \Psi+\hbar \bar{\Psi} \gamma^{\mu} \partial_{\mu} \Psi+m \omega \bar{\Psi} \gamma^{\mu} \delta_{\mu}^{i} \eta \frac{(\boldsymbol{\Theta} \times \mathbf{p})_{i}}{2 \hbar} \Psi+m \omega \bar{\Psi} \frac{(\boldsymbol{\Theta} \times \overleftarrow{\mathbf{p}})_{i}}{2 \hbar} \gamma^{\mu} \eta \delta_{\mu}^{i} \Psi=0 .
$$

We can express it as follows,

$$
\begin{aligned}
0 & =\hbar \partial_{\mu}\left(\bar{\Psi} \gamma^{\mu} \Psi\right)+m \omega \frac{(\boldsymbol{\Theta} \times \mathbf{p})_{i}}{2 \hbar}\left(\bar{\Psi} \gamma^{i} \eta \Psi\right) \\
& =\hbar \partial_{\mu}\left(\bar{\Psi} \gamma^{\mu} \Psi\right)+m \omega \frac{-\mathrm{i} \hbar}{2 \hbar} \epsilon_{i}^{j k} \Theta_{j} \partial_{k}\left(\bar{\Psi} \gamma^{i} \eta \Psi\right) \\
& =\hbar \partial_{\mu}\left(\bar{\Psi} \gamma^{\mu} \Psi\right)-\mathrm{i} \partial_{k}\left(m \omega \frac{1}{2} \epsilon_{i}^{j k} \Theta_{j} \bar{\Psi} \gamma^{i} \eta \Psi\right) \\
& =\hbar \partial_{\mu}\left(\bar{\Psi} \gamma^{\mu} \Psi\right)-\mathrm{i} \partial_{\mu}\left(\delta_{k}^{\mu} m \omega \frac{1}{2} \epsilon_{i}^{j k} \Theta_{j} \bar{\Psi} \gamma^{i} \eta \Psi\right) \\
& =\partial_{\mu}\left(\hbar \bar{\Psi} \gamma^{\mu} \Psi-\mathrm{i} \delta_{k}^{\mu} m \omega \frac{1}{2} \epsilon_{i}^{j k} \Theta_{j} \bar{\Psi} \gamma^{i} \eta \Psi\right) .
\end{aligned}
$$

The conserved current now reads

$$
J_{N C}^{\mu} \equiv \hbar \bar{\Psi} \gamma^{\mu} \Psi-\mathrm{i} \delta_{k}^{\mu} m \omega \frac{1}{2} \epsilon_{i}^{j k} \Theta_{j} \bar{\Psi} \gamma^{i} \eta \Psi .
$$


From this current, we can normalize the wave function, with the fourth component $J_{N C}^{4}$ understood as the probability density. Since the second term in Eq. (53) vanishes when $\mu \neq 1,2,3$, we take

$$
\int d^{3} x(-\mathrm{i}) \hbar \bar{\Psi}(x) \gamma^{4} \Psi(x)=1
$$

that is to say,

$$
\begin{aligned}
& 1=\int d^{3} x(-\mathrm{i}) \hbar\left(\begin{array}{ll}
\psi_{1}^{\dagger} & \psi_{2}^{\dagger}
\end{array}\right)\left(\begin{array}{cc}
0 & -\mathrm{i} \\
\mathrm{i} & 0
\end{array}\right)\left(\begin{array}{cc}
0 & 0 \\
-\sqrt{2} & 0
\end{array}\right)\left(\begin{array}{l}
\psi_{1} \\
\psi_{2}
\end{array}\right) \\
& =\int d^{3} x(-\mathrm{i}) \hbar\left(\begin{array}{ll}
\psi_{1}^{\dagger} & \psi_{2}^{\dagger}
\end{array}\right)\left(\begin{array}{cc}
\mathrm{i} \sqrt{2} & 0 \\
0 & 0
\end{array}\right)\left(\begin{array}{l}
\psi_{1} \\
\psi_{2}
\end{array}\right) \\
& =\int d^{3} x \sqrt{2} \psi_{1}^{\dagger} \psi_{1}
\end{aligned}
$$

We choose

$$
N_{s}^{s_{z}}=N\left(\begin{array}{c}
\left(\begin{array}{c}
v^{+} \\
v^{-}
\end{array}\right) \\
\left(\begin{array}{l}
v^{+} \\
v^{-}
\end{array}\right)
\end{array}\right),
$$

( $N$ is a constant) such that

$$
\Psi=N\left(\begin{array}{l}
\left(\begin{array}{c}
v^{+} \chi_{1}^{+}(r) \\
v^{-} \chi_{1}^{-}(r)
\end{array}\right) \\
\left(\begin{array}{l}
v^{+} \chi_{2}^{+}(r) \\
v^{-} \chi_{2}^{-}(r)
\end{array}\right)
\end{array}\right) e^{\mathrm{i} m_{\theta} \theta}
$$

with

$$
\chi_{s}^{ \pm}(r)=e^{-\frac{1}{2} \frac{M \Xi}{\hbar} r^{2}} r^{\left|m_{\theta}\right|}{ }_{1} F_{1}\left(\alpha, \gamma, \frac{M \Xi}{\hbar} r^{2}\right) .
$$

Since $\alpha=-n$, the confluent hypergeometric function can be identified with the associated Laguerre function,

$$
L_{n}^{\left(\left|m_{\theta}\right|\right)}\left(\frac{M \Xi}{\hbar} r^{2}\right)=\left(\begin{array}{c}
n+\left|m_{\theta}\right| \\
n
\end{array}\right){ }_{1} F_{1}\left(-n,\left|m_{\theta}\right|+1, \frac{M \Xi}{\hbar} r^{2}\right),
$$

that is normalized as

$$
\int d y e^{-y} y^{\left|m_{\theta}\right|} L_{n}^{\left(\left|m_{\theta}\right|\right)}(y) L_{n}^{\left(\left|m_{\theta}\right|\right)}(y)=\frac{\Gamma\left(n+\left|m_{\theta}\right|+1\right)}{n !} .
$$

Therefore, it follows that

$$
\begin{aligned}
1= & \int d^{3} x \sqrt{2} \psi_{1}^{\dagger} \psi_{1}=\int d r r d \theta \int d z \delta(z) \sqrt{2} \psi_{1}^{\dagger} \psi_{1} \\
= & |N|^{2} \sqrt{2} 2 \pi \int d r r\left(\left(v^{+}\right)^{*} v^{+} \xi_{1}^{+}(r) \xi_{1}^{+}(r)+\left(v^{-}\right)^{*} v^{-} \xi_{1}^{-}(r) \xi_{1}^{-}(r)\right) \\
= & |N|^{2} \sqrt{2} 2 \pi \int d r r e^{-\frac{M \Xi}{\hbar} r^{2}} r^{2\left|m_{\theta}\right|} \\
& \times \frac{1}{\left(\begin{array}{c}
n+\left|m_{\theta}\right| \\
n
\end{array}\right)}\left(\left(v^{+}\right)^{*} v^{+}+\left(v^{-}\right)^{*} v^{-}\right)\left[L_{n}^{\left(\left|m_{\theta}\right|\right)}\left(\frac{M \Xi}{\hbar} r^{2}\right)\right]^{2} .
\end{aligned}
$$


Changing the variables

$$
\begin{gathered}
y=\frac{M \Xi}{\hbar} r^{2} \Rightarrow d y=2 \frac{M \Xi}{\hbar} r d r \\
1=|N|^{2} \sqrt{2} \pi\left(\frac{\hbar}{M \Xi}\right)^{\left|m_{\theta}\right|+1} \frac{1}{\left(\begin{array}{c}
n+\left|m_{\theta}\right| \\
n
\end{array}\right)^{2}}\left(\left(v^{+}\right)^{*} v^{+}+\left(v^{-}\right)^{*} v^{-}\right) \\
\times \int d y e^{-y} y^{\left|m_{\theta}\right|}\left[L_{n}^{\left(\left|m_{\theta}\right|\right)}(y)\right]^{2} \\
=|N|^{2} \sqrt{2} \pi\left(\frac{\hbar}{M \Xi}\right)^{\left|m_{\theta}\right|+1} \frac{1}{\left.\left(\begin{array}{c}
\left.n+\left|m_{\theta}\right|\right)^{2} \\
n
\end{array}\right)^{*} v^{+}+\left(v^{-}\right)^{*} v^{-}\right)} \\
\times \frac{\Gamma\left(n+\left|m_{\theta}\right|+1\right)}{n !}
\end{gathered}
$$

This way, we find that the constant $N_{s}^{s_{z}}$ in Eq. (47) is given by

$$
|N|^{2}=\frac{1}{\sqrt{2} \pi\left(\frac{\hbar}{M \Xi}\right)^{\left|m_{\theta}\right|+1}\left(\left(v^{+}\right)^{*} v^{+}+\left(v^{-}\right)^{*} v^{-}\right) \Gamma\left(n+\left|m_{\theta}\right|+1\right)} n !\left(\begin{array}{c}
n+\left|m_{\theta}\right| \\
n
\end{array}\right)^{2}
$$

\section{Concluding Remarks}

We examined the Galilean, or non-relativistic, version of the Dirac oscillator for spin-half fields in a non-commutative space. We employed the Galilean covariant approach (with equations projected from a $(4,1)$ space-time with light-cone coordinates to a $(3,1)$ Galilean space-time) to obtain the wave equation. We found the wave functions and their energy levels in the plane.

The energy spectrum is described by Eq. (50), with its commutative limit given in Eq. (51). If we take the limit $\Omega \rightarrow 0$, that is, only the momentum-momentum commutators vanish, then Eq. (50) reduces to

$$
\begin{aligned}
E_{n, m_{\theta}, s, s_{z}}= & 2\left(2 n+\left|m_{\theta}\right|+1\right) \hbar \sqrt{\frac{\omega^{2}}{4}\left(1+\frac{m^{2} \omega^{2} \Theta^{2}}{4 \hbar^{2}}\right)+\frac{s s_{z} \omega \Theta}{\hbar}} \\
& +\hbar m_{\theta}\left(s s_{z} \omega-\frac{m \omega^{2} \Theta}{2 \hbar}\right)+\frac{3}{2} \hbar s \omega-\frac{m s_{z} \omega^{2} \Theta}{2}
\end{aligned}
$$

which depends on the space-space non-commutativity parameter $\Theta$. An example of spacespace non-commutativity is the study of the hydrogen atom with the Dirac equation in Ref. [38].

As a continuation of this work and Ref. [1], we are currently working on the noncommutative Galilean DKP oscillator for spin-one particles with external magnetic fields. Since there exist two Galilean limits of electromagnetism [22], the relation between noncommutativity and magnetic fields $[39,40]$ should take a different guise in the Galilean context than in the relativistic context. Relativistic spin-one DKP theory in the presence of external electromagnetic fields has been studied in Ref. [41]. Spin-one DKP equation with non-commutative geometry has been investigated in Refs. [42-44]. 
Acknowledgements We acknowledge partial support by the Natural Science and Engineering Research Council (NSERC) of Canada (MdeM) and Coordenação de Aperfeiçoamento de Pessoal de Nível Superior (CAPES) of Brazil (ESS).

\section{Appendix}

Proof of Eq. (22)

$$
\begin{aligned}
(\sigma \cdot \hat{\mathbf{p}})(\sigma \cdot \hat{\mathbf{p}}) & =\frac{1}{2}\left(\sigma_{i} \sigma_{j} \hat{p}_{i} \hat{p}_{j}-\sigma_{i} \sigma_{j} \hat{p}_{j} \hat{p}_{i}+2 \delta_{i j} \hat{p}_{j} \hat{p}_{i}\right) \\
& =\frac{1}{4}\left[\sigma_{i}, \sigma_{j}\right]\left(\hat{p}_{i} \hat{p}_{j}-\hat{p}_{j} \hat{p}_{i}\right)+\hat{\mathbf{p}} \cdot \hat{\mathbf{p}} \\
& =-\epsilon_{i j k} \epsilon_{i j l} S_{k} \Omega_{l}+\mathbf{p}^{2}-\frac{1}{\hbar} \boldsymbol{\Omega} \cdot(\mathbf{r} \times \mathbf{p})+\frac{1}{4 \hbar^{2}}(\boldsymbol{\Omega} \times \mathbf{r})^{2} \\
& =-\frac{2}{\hbar} \mathbf{S} \cdot \boldsymbol{\Omega}+\mathbf{p}^{2}-\frac{1}{\hbar} \boldsymbol{\Omega} \cdot \mathbf{L}+\frac{1}{4 \hbar^{2}}(\boldsymbol{\Omega} \times \mathbf{r})^{2} .
\end{aligned}
$$

We obtain Eq. (23) in the same way.

Proof of Eq. (24)

$$
\begin{aligned}
&(\sigma \cdot \hat{\mathbf{r}})(\sigma \cdot \hat{\mathbf{p}})-(\sigma \cdot \hat{\mathbf{p}})(\sigma \cdot \hat{\mathbf{r}}) \\
&=\sigma_{i} \sigma_{j} \hat{r}_{i} \hat{p}_{j}+\sigma_{i} \sigma_{j} \hat{p}_{j} \hat{r}_{i}-2 \delta_{i j} \hat{p}_{j} \hat{r}_{i} \\
&= 2 \sigma_{i} \sigma_{j} \hat{r}_{i} \hat{p}_{j}-\mathrm{i} \hbar \sigma_{i} \sigma_{j} \Delta_{i j}-2 \hat{\mathbf{p}} \cdot \hat{\mathbf{r}} \\
&= 2 \sigma_{i} \sigma_{j} \hat{r}_{i} \hat{p}_{j}-\mathrm{i} \hbar \sigma_{i} \sigma_{j}\left[\left(1+\frac{\boldsymbol{\Theta} \cdot \boldsymbol{\Omega}}{4 \hbar^{2}}\right) \delta_{i j}-\frac{\Omega_{i} \Theta j}{4 \hbar^{2}}\right]-2 \hat{\mathbf{p}} \cdot \hat{\mathbf{r}} \\
&= 2 \sigma_{i} \sigma_{j} \hat{r}_{i} \hat{p}_{j}-\mathrm{i} \hbar \delta_{i j}\left(1+\frac{\boldsymbol{\Theta} \cdot \boldsymbol{\Omega}}{4 \hbar^{2}}\right) \delta_{i j}+\mathrm{i} \hbar \frac{(\sigma \cdot \boldsymbol{\Omega})(\sigma \cdot \boldsymbol{\Theta})}{4 \hbar^{2}}-2 \hat{\mathbf{p}} \cdot \hat{\mathbf{r}} \\
&= 2 \sum_{i \neq j} \sigma_{i} \sigma_{j} \hat{r}_{i} \hat{p}_{j}-3 \mathrm{i} \hbar+\mathrm{i} \hbar \frac{(\sigma \cdot \boldsymbol{\Omega})(\sigma \cdot \boldsymbol{\Theta})-3 \boldsymbol{\Theta} \cdot \boldsymbol{\Omega}}{4 \hbar^{2}}+2(\hat{\mathbf{r}} \cdot \hat{\mathbf{p}}-\hat{\mathbf{p}} \cdot \hat{\mathbf{r}}) \\
&= 2 \frac{\left[\sigma_{i}, \sigma_{j}\right]}{2} \hat{r}_{i} \hat{p}_{j}-3 \mathrm{i} \hbar+\mathrm{i} \frac{\mathrm{i} \sigma \cdot(\boldsymbol{\Omega} \times \boldsymbol{\Theta})-2 \boldsymbol{\Theta} \cdot \boldsymbol{\Omega}}{4 \hbar}+2 \mathrm{i} \hbar \Delta_{i i} \\
&= \frac{4 \mathrm{i}}{\hbar} \epsilon_{i j k} S_{k} \hat{r}_{i} \hat{p}_{j}-3 \mathrm{i} \hbar+\mathrm{i} \frac{\mathrm{i} \sigma \cdot(\boldsymbol{\Omega} \times \boldsymbol{\Theta})-2 \boldsymbol{\Theta} \cdot \boldsymbol{\Omega}}{4 \hbar}+2 \mathrm{i} \hbar\left[3\left(1+\frac{\boldsymbol{\Theta} \cdot \boldsymbol{\Omega}}{4 \hbar^{2}}\right)-\frac{\boldsymbol{\Omega} \cdot \boldsymbol{\Theta}}{4 \hbar^{2}}\right] \\
&= \frac{4 \mathrm{i}}{\hbar} \mathbf{S} \cdot(\hat{\mathbf{r}} \times \hat{\mathbf{p}})+3 \mathrm{i} \hbar+\mathrm{i} \frac{\mathrm{i} \sigma \cdot(\boldsymbol{\Omega} \times \boldsymbol{\Theta})+2 \boldsymbol{\Theta} \cdot \boldsymbol{\Omega}}{4 \hbar} \\
&= 4 \mathrm{i} \mathbf{S} \cdot(\mathbf{r} \times \mathbf{p})+\frac{2 \mathrm{i}}{\hbar^{2}} \mathbf{S} \cdot[\mathbf{r} \times(\boldsymbol{\Omega} \times \mathbf{r})]+\frac{2 \mathrm{i}}{\hbar^{2}} \mathbf{S} \cdot[(\boldsymbol{\Theta} \times \mathbf{p}) \times \mathbf{p}] \\
&-\frac{\mathrm{i}}{\hbar^{3}} \mathbf{S} \cdot[(\boldsymbol{\Theta} \times \mathbf{p}) \times(\boldsymbol{\Omega} \times \mathbf{r})]+3 \mathrm{i} \hbar+\mathrm{i} \frac{\mathrm{i} \sigma \cdot(\boldsymbol{\Omega} \times \boldsymbol{\Theta})+2 \boldsymbol{\Theta} \cdot \boldsymbol{\Omega}}{4 \hbar} \\
&= \frac{4 \mathrm{i}}{\hbar} \mathbf{S} \cdot \mathbf{L}-\frac{2 \mathrm{i}}{\hbar^{2}}(\mathbf{S} \times \mathbf{r}) \cdot(\boldsymbol{\Omega} \times \mathbf{r})+\frac{2 \mathrm{i}}{\hbar^{2}}(\mathbf{S} \times \mathbf{p}) \cdot(\boldsymbol{\Theta} \times \mathbf{p}) \\
&-\frac{\mathrm{i}}{\hbar^{3}} \mathbf{S} \cdot[(\boldsymbol{\Theta} \times \mathbf{p}) \times(\boldsymbol{\Omega} \times \mathbf{r})]+3 \mathrm{i} \hbar+\mathrm{i} \frac{\mathrm{i} \sigma \cdot(\boldsymbol{\Omega} \times \boldsymbol{\Theta})+2 \boldsymbol{\Theta} \cdot \boldsymbol{\Omega}}{4 \hbar} .
\end{aligned}
$$


The last line contains the following term,

$$
(\boldsymbol{\Theta} \times \mathbf{p}) \times(\boldsymbol{\Omega} \times \mathbf{r})=-\boldsymbol{\Omega}(\boldsymbol{\Theta} \cdot \mathbf{L})-[\boldsymbol{\Theta} \cdot(\mathbf{p} \times \boldsymbol{\Omega})] \mathbf{r},
$$

so that we obtain

$$
\begin{aligned}
(\sigma \cdot \hat{\mathbf{r}})(\sigma \cdot \hat{\mathbf{p}})-(\sigma \cdot \hat{\mathbf{p}})(\sigma \cdot \hat{\mathbf{r}})= & \frac{4 \mathrm{i}}{\hbar} \mathbf{S} \cdot \mathbf{L}-\frac{2 \mathrm{i}}{\hbar^{2}}(\mathbf{S} \times \mathbf{r}) \cdot(\boldsymbol{\Omega} \times \mathbf{r})+\frac{2 \mathrm{i}}{\hbar^{2}}(\mathbf{S} \times \mathbf{p}) \cdot(\boldsymbol{\Theta} \times \mathbf{p}) \\
& +\frac{\mathrm{i}}{\hbar^{3}}[(\mathbf{S} \cdot \boldsymbol{\Omega})(\boldsymbol{\Theta} \cdot \mathbf{L})+[\boldsymbol{\Theta} \cdot(\mathbf{p} \times \boldsymbol{\Omega})](\mathbf{S} \cdot \mathbf{r})]+3 \mathrm{i} \hbar \\
& +\mathrm{i} \frac{\mathrm{i} \sigma \cdot(\boldsymbol{\Omega} \times \boldsymbol{\Theta})+2 \boldsymbol{\Theta} \cdot \boldsymbol{\Omega}}{4 \hbar} .
\end{aligned}
$$

\section{References}

1. de Melo, G.R., de Montigny, M., Santos, E.S.: Int. J. Theor. Phys. 51, 2524 (2012)

2. Petiau, G.: Académie Royale de Belgique, Classe des Sciences, Mémoires, Collection (8) 16(2), 1 (1936)

3. Duffin, R.J.: Phys. Rev. 54, 1114 (1938)

4. Kemmer, N.: Proc. R. Soc. A 166, 127 (1938)

5. Kemmer, N.: Proc. R. Soc. A 173, 91 (1939)

6. Fischbach, E., Louck, J.D., Nieto, M.M., Scott, C.K.: J. Math. Phys. 15, 60 (1974) (and references therein)

7. Vijayalakshmi, B., Seetharaman, M., Mathews, P.M.: J. Phys. A, Math. Gen. 12, 665 (1979)

8. Guertin, R.F., Wilson, T.L.: Phys. Rev. D. 15, 1518 (1977)

9. Dirac, P.A.M.: Proc. R. Soc. A 117, 610 (1928)

10. Peskin, M., Schroeder, D.: An Introduction to Quantum Field Theory. Perseus Books, New York (1995)

11. Ryder, L.H.: Quantum Field Theory, 2nd edn. Cambridge University Press, Cambridge (1993)

12. Snyder, H.S.: Phys. Rev. 71, 38 (1947)

13. Bargmann, V.: Ann. Math. 59, 1 (1954)

14. Lévy-Leblond, J.M.: J. Math. Phys. 4, 776 (1963)

15. Brennich, R.H.: Ann. Inst. Henri Poincaré 13, 221 (1970)

16. Lévy-Leblond, J.M.: In: Loebl, E.M. (ed.) Group Theory and Applications, vol. 2, p. 221. Academic Press, New York (1971)

17. de Montigny, M., Niederle, J., Nikitin, A.G.: J. Phys. A, Math. Gen. 39, 9365 (2006)

18. Havas, P.: Rev. Mod. Phys. 36, 938 (1964)

19. Takahashi, Y.: Fortschr. Phys. 36, 63 (1988)

20. Takahashi, Y.: Fortschr. Phys. 36, 83 (1988)

21. Omote, M., Kamefuchi, S., Takahashi, Y., Ohnuki, Y.: Fortschr. Phys. 37, 933 (1989)

22. Le Bellac, M., Lévy-Leblond, J.M.: Nuovo Cimento B 14, 217 (1973)

23. Cai, S., Jing, T., Guo, G., Zhang, R.: Int. J. Theor. Phys. 49, 1699 (2010)

24. Lukierski, J., Stichel, P.C., Zakrzewski, W.J.: Ann. Phys. 260, 224249 (1997)

25. Duval, C., Horváthy, P.A.: J. Phys. A, Math. Gen. 34, 10097 (2001)

26. Horváthy, P.A., Martina, L., Stichel, P.C.: Phys. Lett. B 564, 149 (2003)

27. Horváthy, P.A., Martina, L., Stichel, P.C.: Nucl. Phys. B 673, 301 (2003)

28. Horváthy, P.A.: Talk given at the Int. Conf. Noncommutative Geometry and Quantum Physics, Kolkata 2006. Available at arXiv:hep-th/0602133

29. Horváthy, P.A., Martina, L., Stichel, P.C.: Symmetry, integrability and geometry: methods and applications. SIGMA 6, 060 (2010)

30. Curtright, T., Fairlie, D., Zachos, C.: Phys. Rev. D 58, 025002 (1998)

31. Lévy-Leblond, J.M.: Commun. Math. Phys. 6, 286 (1967)

32. Santos, E.S., de Montigny, M., Khanna, F.C., Santana, A.E.: J. Phys. A, Math. Gen. 37, 9771 (2004)

33. Santos, E.S., de Melo, G.R.: Int. J. Theor. Phys. 50, 332 (2011)

34. Moshinsky, M., Szczepaniak, A.: J. Phys. A, Math. Gen. 22, L817 (1989)

35. Horváthy, P.A., Martina, L., Stichel, P.C.: Symmetry, integrability and geometry: methods and applications. SIGMA 6, 060 (2010)

36. de Montigny, M., Khanna, F.C., Santana, A.E., Santos, E.S.: J. Phys. A, Math. Gen. 34, 8901 (2001)

37. Abramowitz, M., Stegun, I.A.: Handbook of Mathematical Functions. Dover, New York (1972)

38. Adorno, T.C., Baldiotti, M.C., Chaichian, M., Gitman, D.M., Tureanu, A.: Phys. Lett. B 682, 235 (2009) 
39. Lukierski, J., Stichel, P.C., Zakrzewski, W.J.: Phys. Lett. B 602, 249 (2004)

40. Delduc, F., Duret, Q., Gieres, F., Lefrançois, M.: J. Phys. Conf. Ser. 103, 012020 (2008)

41. Vijayalakshimi, B., Seetharaman, M., Mathews, P.M.: J. Phys. A, Math. Gen. 12, 665 (1979)

42. Falek, M., Merad, M.: Commun. Theor. Phys. 50, 587 (2008)

43. Falek, M., Merad, M.: J. Math. Phys. 50, 023508 (2009)

44. Falek, M., Merad, M.: J. Math. Phys. 51, 033516 (2010) 